\title{
Clinical relevance of persistent postoperative pain after total hip replacement - a prospective observational cohort study
}

This article was published in the following Dove Press journal:

Journal of Pain Research

7 September 2017

Number of times this article has been viewed

\author{
Joachim Erlenwein ${ }^{1, *}$ \\ Martin Müller ${ }^{1, *}$ \\ Deborah Falla ${ }^{1,2}$ \\ Michael Przemeck ${ }^{3}$ \\ Michael Pfingsten' \\ Stefan Budde ${ }^{4}$ \\ Michael Quintel' \\ Frank Petzke'
}

'Department of Anesthesiology, Pain Clinic, University Medical Center Göttingen, Georg August University of Göttingen, Göttingen, Germany; ${ }^{2}$ Centre of Precision Rehabilitation for Spinal Pain (CPR Spine), School of Sport, Exercise and Rehabilitation Sciences, College of Life and Environmental Sciences, University of Birmingham, Birmingham, UK; ${ }^{3}$ Department of Anesthesiology and Intensive Care, Annastift, Hannover, ${ }^{4}$ Department of Orthopedic Surgery, Medical School Hannover, Hannover, Germany

*These authors contributed equally to this work
Correspondence: Joachim Erlenwein Department of Anesthesiology, Pain Clinic, University Medical Center, Georg August University of Göttingen, RobertKoch-Str. 40, 37075 Göttingen, Germany $\mathrm{Tel}+4955 \mathrm{I} 398816$

$\mathrm{Fax}+49551394164$

Email joachim.erlenwein@med.unigoettingen.de
Purpose: The development of persistent postoperative pain may occur following surgery, including total hip replacement. Yet, the prevalence may depend on the definition of persistent pain. This observational cohort study explored whether the prevalence of persistent pain after total hip replacement differs depending on the definition of persistent pain and evaluated the impact of ongoing pain on the patient's quality of life 6 months after surgery.

Patients and methods: Pre- and postoperative characteristics of 125 patients undergoing elective total hip replacement were assessed and 104 patients were available for the follow-up interview, 6 months after surgery.

Results: Six months after surgery, between $26 \%$ and $58 \%$ of patients still reported hip pain depending on the definition of persistent pain. Patients with moderate-to-severe persistent pain intensity ( $>3$ on a numerical rating scale) were more restricted in their daily life activities (Chronic Pain Grade - disability score) but did not differ in reported quality of life (Short-Form 12) from those with no pain or milder pain intensity. Maximal preoperative pain intensity and body mass index were the only independent factors influencing daily function 6 months after total hip replacement.

Conclusion: These findings support a high prevalence of persistent postoperative pain after total hip replacement and a large variability depending on the definition used. There was a close relation between physical functioning and pain as well as relevance of the patient's psychological state at the time of the operation.

Keywords: chronic postoperative pain, acute pain management, hip replacement, quality of life, German Pain Questionnaire

\section{Introduction}

The development of chronic postoperative pain has been described for several surgical procedures. ${ }^{1}$ Considering the large number of surgical procedures performed every year in Western industrialized countries, the prevention of chronic postoperative pain is currently a significant focus in acute pain management. ${ }^{2,3}$ The incidence of postoperative pain varies depending on the procedure and individual surgical, psychological, genetic, and social risk factors. ${ }^{4} \mathrm{Up}$ to $65 \%$ of patients undergoing thoracic and cardiac surgery complain of chronic postoperative pain. ${ }^{1,5}$ Breast surgery and amputations are also associated with a high prevalence of postoperative pain. ${ }^{1,6}$

However, the prevalence of this pain may vary depending on the definition used and the timeframe after surgery. ${ }^{7-9}$ Some authors define any pain as chronic, while others consider pain only defined by a minimum intensity (e.g., $>3$ on the numeric rating scale $[\mathrm{NRS}]$ ), and some require a clear differentiation from pre-existing pain before surgery. ${ }^{7-10}$ 
Particularly for orthopedic procedures, which are typically performed for a pre-existing painful condition, the distinction between pre-existing and chronic postoperative pain complicates a clear differentiation. Nevertheless, chronic or persistent postoperative pain also affects a relevant proportion of orthopedic patients. One of the most frequently conducted orthopedic surgical procedures in industrialized countries is total hip replacement. The reported prevalence of persistent postoperative pain after total hip replacement ranged between $27 \%$ and $38 \%$ of the patients, depending on the definition used. ${ }^{11-14}$ Such persistent pain likely has a relevant impact on the patient's activities of daily life and their quality of life, which is a serious matter of suffering in addition to pain. ${ }^{15-17}$

The aim of this prospective observational cohort study was to investigate the prevalence of persistent pain after total hip replacement and its impact on patient's quality of life 6 months after surgery. The primary outcome was pain intensity 6 months after surgery. Secondary outcomes were impairment in daily function, life quality, and psychological distress after 6 months. Since a uniform definition of persistent postoperative pain is not currently available, we also explored the prevalence of persistent pain per different definitions (maximum pain intensity in the follow-up NRS $>0$ and maximum pain intensity NRS $>3$ ), already reported in current literature.

\section{Patients and methods}

\section{Patients}

Patients undergoing total hip replacement because of osteoarthritis were invited to participate in the study. Patients were recruited between July and October 2012 at the Orthopedic department of the Medical School Hannover, Germany. Participants gave written informed consent for this study. Patients had to be at least 18 years old and have sufficient knowledge of the German language to understand the study information and the required questionnaire package. Only patients undergoing primary endoprostethic surgery of the hip were included. Exclusion criteria were a neuraxial or regional anesthesia, history of drug abuse, and acute hip pain caused by necrosis of the femoral head. In the occurrence of postoperative delirium syndrome, the patients were excluded. Exclusion criteria for the follow-up interview were the occurrence of surgical complications (e.g., periprosthetic fracture or prostheses loosening).

\section{Protocol}

The protocol was approved by the Ethics committees of the University Medical Center Göttingen (No 5/4/12) and the Medical School Hannover (No 1483-2012) and conducted per the recommendations of the Declaration of Helsinki. Perioperative data of this cohort have been published previously. ${ }^{18}$

\section{Study flow}

Baseline data were assessed on the day before surgery, following admission to the hospital.

Perioperatively, all patients were treated following a standardized pain management protocol established and certified at the institution for several years. ${ }^{19}$

A follow-up was conducted 6 months ( \pm 1 week) after surgery. Patients were interviewed by a study physician (M.M.) via a telephone interview and according to a standardized protocol. Figure 1 provides an overview of participant recruitment and retention. All assessments were conducted using validated German versions. In addition to the description below, Supplementary material provides an overview about the properties of variables and tests. The primary outcome was hip pain intensity 6 months after surgery. Secondary outcomes were impairment in daily life activities, life quality, and psychological distress after 6 months.

\section{Pain characteristics at baseline preoperatively}

The patients' pain history was assessed by two physicians (J.E., A.D.; see Acknowledgments) using a standardized protocol on the basis of a German Pain Questionnaire including localization, duration, pain intensity, and the temporal aspects of pain of all pain sites and analgesic consumption and other chronic pain entities (e.g., low back pain, chronic headache; with at least 6 months duration). ${ }^{20}$

Owing to variable preoperative analgesic use (patients given different nonopioids and rarely opioids), the Medication Quantification Scale (MQS) was used to assess the preoperative analgesic consumption. ${ }^{21,22}$ This scale is a reliable and validated method for quantifying medication use in patients with chronic pain. It is calculated for each preoperative analgesic on the basis of weights assigned by medication class (acetaminophen 2.2, cox-2 inhibitors 2.3, dipyrone [metamizole] 2.3, and diclofenac and ibuprofen and indomethacin 3.4) and dosage level (level $1=$ subtherapeutic dosage and/or on demand, level $2=<50 \%$ of the daily dosage, level $3>50 \%$, and level $4=$ overdose). These scores were summed to provide a quantitative index of total analgesic usage suitable for statistical analyses.

Patients completed a series of standardized questionnaires. To assess the overall severity of chronic pain, based on pain intensity and pain related dysfunction (CPG, Chronic Pain Grade, von Korff), patients were categorized into one 
$\mathrm{n}=172$

Patients were scheduled for total hip replacement $\mathrm{n}=34$ ineligible

$\mathrm{n}=13$ declined to participate

$\mathrm{n}=125$

Complete data collection during the preand perioperative period

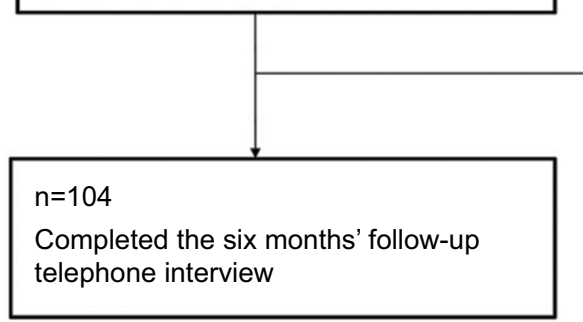

$\mathrm{n}=14$ lost to follow up

$n=4$ refused to continue the study

$\mathrm{n}=3$ removed for medical conditions

Figure I Flow-chart of the study.

of five levels (grade 0: no pain-grade 4: high disability/ severely limiting). ${ }^{23}$ The questionnaire consists of seven items: pain intensity right now, worst pain intensity over the last 3 months, average pain intensity over the last 3 months, limitation in daily activities because of pain, recreational, social, and family activities, limitation in the ability to work because of pain within the last 3 months, and the number of disability days within the last 3 months. Items are scored on an 11-point Likert scale (0-10). Three subscales are calculated, "Pain Intensity Score" is calculated as a mean of reported current, worst, and average pain intensity (ranges from 0 to 100), "Disability points" as a mean of limitations in performing daily life, social, and work activities (ranges from 0 to 100). ${ }^{20}$ These disability points range from " 0 " to " 6 " points (" 0 " =no limitations to " 6 " points $=$ no normal living possible). Finally, the "Disability Points Score" is deduced from a combination of ranked categories of the number of the disability days and the disability score (ranges from 0 to 3 ).

Patients were also classified with the Mainz Pain Staging System (MPSS) to classify the chronicity of the patient's condition (stage 1-3). ${ }^{24}$ The MPSS was assessed as per the reported pain characteristics, the current analgesic regimen, and previous pain-related treatments.

Preoperative hip pain intensity was assessed on the day prior surgery with an 11-point NRS ("0"=no pain to "10"=worst pain). Furthermore, the maximum and the average pain intensities over the last 3 months were obtained using the same scale.
Neuropathic characteristics of the hip pain were assessed with the painDETECT questionnaire. ${ }^{25}$ This questionnaire quantifies typical symptoms of neuropathic pain. The resulting score ranges from 0 to 38 with three categories: $0-12$ points (an unlikely neuropathic component, probability $<15 \%$ ), 13-18 points (unclear result), and 19-38 points (a probable neuropathic component, probability $>90 \%$ ). The absolute score was used for further analyses.

\section{Anesthesia procedure}

All patients received $20-30 \mathrm{mg}$ dipotassium chlorazepat in the evening and the morning prior to the surgical procedure as premedication. General anesthesia was induced by remifentanil (1-1.5 $\mu \mathrm{g} / \mathrm{kg}$ body weight [bw] $/ 3 \mathrm{~min}$ ) and propofol (1-2 $\mathrm{mg} / \mathrm{kg} \mathrm{bw}$ ), and orotracheal intubation was facilitated by $0.5 \mathrm{mg} / \mathrm{kg}$ bw atracurium. Anesthesia was maintained by sevoflurane 0.7-1.0 MAC or propofol 3.5-4.5 mg/kg bw/h, along with Remifentanil $0.15-0.25 \mu \mathrm{g} / \mathrm{kg} / \mathrm{min}$, and was monitored by EEG.

During the final stage of the operation, patients received piritramide $(0.1 \mathrm{mg} / \mathrm{kg} \mathrm{bw})$ and metamizole $(15 \mathrm{mg} / \mathrm{kg}$ bw, if contraindicated, equal amount paracetamol) intravenously.

\section{Protocol for postoperative analgesia}

The postoperative analgesia was standardized on the basis of a protocol for adjusting the analgesic level: immediately after surgery in the recovery area, patients received a 10-20 mg slow-release oxycodone per os (p.o.) (age and/or weight 
adapted: $10 \mathrm{mg}$ if weight $<60 \mathrm{~kg}$ and/or age $>70$ years, all others $20 \mathrm{mg}$ ) and $600 \mathrm{mg}$ ibuprofen p.o. In the recovery area, intravenous piritramide was titrated until the intensity was $\leq 3$ on the NRS. In the ward, oral slow-release oxycodone (1-0-1) and ibuprofen (1-1-1) started in the recovery area were continued for the following days and adapted according to pain intensity following an algorithm on the basis of routine pain measurement. If the NRS was $>3$, patients were offered 1.3-2.6 mg hydromorphone p.o. (age and weight adjusted). If the NRS remained $>3$ after $60 \mathrm{~min}$, a second dose was offered. If hydromorphone was necessary three times within $24 \mathrm{~h}$, slow-release oxycodone was increased. If the patients' chart revealed that the pain intensity was $<3$ on the NRS over the past $24 \mathrm{~h}$, slow-release oxycodone was reduced step wise.

\section{Postoperative pain intensity and analgesic consumption}

Intensity of pain on movement and maximum perceived postoperative pain were recorded separately on the first, third, fifth, and seventh postoperative day using the respective subscales from the German outcome "QUIPS"-questionnaire (quality improvement in postoperative pain management [Qualitätsverbesserung in der postoperativen Schmerztherapie]), which is validated for postoperative pain assessment. ${ }^{26,27}$ The intensity of postoperative pain was assessed on the 11-point NRS. Opioid consumption was recorded in morphine equivalent (ME) in $\mathrm{mg}$ - for the first $48 \mathrm{~h}$ after surgery and the third, fifth, and seventh postoperative day (total daily dose, conversion factor to morphine: oxycodone 0.75 , hydromorphone 0.13 , piritramid 1.5 , fentanyl 0.01 , tilidin/tramadol 10, buprenorphine 0.03 , and intravenous vs oral morphine $3: 1$ ).

\section{Quality of life}

Health-related quality of life was assessed with the ShortForm 12 (SF 12) Health Survey to measure functional health and well-being from the patient's point of view. ${ }^{28}$ It has a validated German version and is a brief and reliable measure of overall health status (summarized as physical health and mental health component). It has been extensively used in large population health surveys. Higher scores indicate better general health.

\section{Psychological symptoms and fear of movement}

Psychological distress was assessed using the German version of the validated Depression Anxiety and Stress Scale
(DASS). ${ }^{29}$ It includes a set of three scales designed to measure the self-reported negative emotional states of depression, anxiety, and stress. Each scale contains seven items and scores are calculated by summing item scores. Relevant cutoffs for depression and stress are ten points or more, and for anxiety, six points or more..$^{20}$

Pain-related fear of movement was assessed using the German version of the Tampa Scale for kinesiophobia (TSK), which is a reliable and valid self-report et al to assess kinesiophobia, or fear of movement and/or (re)injury which have been confirmed as important predictors for the persistence of pain-related disability. ${ }^{30,31}$ Results are calculated by summing the items (total sum score ranging between 17 and 68). A high value indicates a high degree of kinesiophobia (a cutoff from 38 points or more is defined as a clinically relevant fear of movement).

\section{Cognitive appraisal of pain, somatization, and catastrophizing}

The Patient Health Questionnaire (PHQ) is a tool to establish provisional diagnoses for selected DSM-IV disorders. ${ }^{30} \mathrm{It}$ includes a scale to assess somatization (PHQ-15). Reliability and validity of the PHQ-15 are high in clinical and occupational health care settings, and it has been validated for use in German. Patients report the severity of 15 symptoms over the last 4 weeks as 0 ("not bothered at all"), 1 ("bothered a little"), or 2 ("bothered a lot"). The total PHQ-15 score ranges from 0 to 30 ( $\geq 5$ mild, $\geq 10$ moderate, and $\geq 15$ severe levels of somatization).

Cognitive appraisal of pain and catastrophizing were assessed using the Kiel Pain Inventory (KPI), which is a wellestablished validated tool in German to assess various aspects of chronic pain within cross-sectional as well as prospective studies. ${ }^{31}$ It includes three subscales: The Catastrophizing Thoughts Scale (CTS) consists of 5 items describing the threatening aspects of pain (e.g., "What will happen if the pain gets worse?"). The Thoughts of Help-/Hopelessness Scale (THS) consists of 9 items that focus on lack of hope and impossibility to become pain free (e.g., "It's not going to get any better"). The Thought Suppression Scale (TSS) consists of four items (e.g., "Pull yourself together!", "Don't make such a fuss!"). It describes attempts to suppress painsensations, pain-related emotions, and thoughts.

Patients indicate the extent to which they have experienced those thoughts in the past 14 days when they experienced pain on a 7-point Likert scale (0 "never", 6 "always"). For each scale, results are summed and divided by the number of items for each scale. Higher scores indicate 
a higher occurrence of catastrophizing, help-/hopelessness, and suppressive thoughts.

\section{Preoperative quantitative sensory tests}

As an assessment of overall pain sensitivity, the pressure pain threshold (PPT) was measured using an electronic pressure algometer (Somedic Production, Stockholm, Sweden) bilaterally over five sites: the thumb, lateral epicondylus, upper division of the trapezius, quadriceps femoris, and tibialis anterior. The algometers' probe tip $\left(1 \mathrm{~cm}^{2}\right)$ was applied to each site. Patients were advised to indicate when they perceived pain for the first time during pressure stimulation with slowly increasing intensity $(50 \mathrm{kPa} / \mathrm{s})$. Pressure stimulation stopped at the patients' report of pain or when maximum pressure intensity $(1000 \mathrm{kPa})$ was reached. The mean threshold over all 10 testing sites was calculated for further analyses.

The Conditioned Pain Modulation (CPM) was measured to quantify the ability of a conditioning stimulus on a second pain stimulus (pain inhibiting pain). ${ }^{34,35}$ As a test stimulus, the PPT was assessed repetitively (30 s) with the pressure algometer on the thumb of the dominant hand.

The conditioning pain stimulus consisted of submersion of the nondominant hand in a hot water bath with a temperature of $45^{\circ} \mathrm{C}$ (Thermostat, Julabo Labortechnik, Seelbach, Germany). The test stimulus was measured three times, before the water bath started and three times while the conditioning stimulus (water bath) was applied. The ratio of the mean of the three measurements of the PPT during the conditioned pain stimulus (hot water) and the mean of the baseline measurement (test stimuli) was calculated and defined as CPM (ratio PPT thumb hot water bath/PPT thumb baseline). Measurements were performed by two research assistants who were trained prior to the study in approximately equal shares (St.B., M.G.; see acknowledgement).

\section{Outcome parameters at the 6-month follow-up}

Outcome parameters at the 6-month follow-up were recorded via telephone interview (M.M.). The intensity of persistent postoperative pain was also measured with an 11-point NRS. Patients were asked for the maximum hip pain during the last four weeks prior to the interview and the intensity of hip pain at the time of the interview.

To evaluate the importance of the definition of persistent pain on prevalence rates, we considered different definitions: pain within the last 4 weeks (maximum pain intensity NRS $>0$ ), "moderate" to "severe" pain within the last 4 weeks (maximum pain intensity NRS $>3$ ), and current pain at the time of the interview (NRS $>0$ ).

Moreover, we evaluated the impact of persistent postoperative pain on the patients' quality of life (SF 12), functionality (disability score of the CPG), and psychological distress (DASS and TSK).

\section{Statistical analyses}

The analyses were performed with SPSS (IBM SPSS Statistics for Windows, Version 22.0., IBM Corporation, Armonk, NY, USA) and STATISTICA (StatSoft Ver. 12.7, Tulsa, OK, USA).

Ordinal variables were presented using the median (median [1-3 Quartile]). Metric variables were presented using the arithmetic mean and the SD (mean \pm SD). Categorical variables were presented in percent and the absolute number of patients. Percentages were rounded.

To compare the characteristics of patients with the definition "no" and "mild" persistent postoperative pain vs patients with "moderate" to "severe" pain intensity, patients were separated into two groups on the basis of their maximum hip pain intensity during the last 4 weeks before the telephone interview 6 months after surgery (NRS $\leq 3$ vs NRS $>3$ ).

On account of the non-normal distribution of most variables, these group comparisons were performed by a nonparametric test (Mann-Whitney $U$-test). The distribution of frequencies of dichotomous variables in groups was described using the Pearson $\chi^{2}$ test. The level of significance was set as $p<0.05$.

To analyze the association between patient characteristics and poor functional outcome after 6 months (disability score of the CPG), univariate analyses (logistic ordinal regression) were performed as a first step with all perioperative variables: age (years), sex, body mass index (BMI) $\left(\mathrm{kg} / \mathrm{m}^{2}\right)$, MPSS, quality of life (SF 12), maximum hip pain before surgery (NRS), severity of chronic pain (CPG), preoperative analgesics (MQS), psychological distress (DASS - depression, anxiety, stress), kinesiophobia (TSK), catastrophizing (CTS), helplessness (THS), thought suppression (TSS), somatization (PHQ), preoperative neuropathic component of hip pain (painDETECT), pain sensitivity (PPT, $\mathrm{kPa}$ ), and pain inhibition (CPM).

To reduce the number of variables, the cumulative opioid consumption, postoperative pain on movement, and maximum pain were calculated by summing the respective scores (mg ME, NRS) over days 1, 3, 5, and 7. Variables that correlated significantly with the disability score $(p<0.05 ; \mathrm{CPG})$ were analyzed in a second step in a multivariate analyzes model. 


\section{Results}

A total of 172 patients scheduled for elective total hip replacement were screened for recruitment (Figure 1). Of these, 47 patients did not meet the inclusion criteria or were excluded during the initial perioperative assessment, (primary exclusions: three took part in other studies, one was younger than 18 years, one did not speak German, three were hospitalized outside of clinical routine and could not be measured preoperatively, one was an active drug user, one had dementia, nine had neuroaxial anesthesia, fifteen had their surgery postponed and thirteen patients declined to participate) resulting in 125 patients with complete perioperative data.

A total of 104 patients were included into the analyses for the 6-month follow-up. Patients' characteristics and preoperative baseline parameters are presented in Table 1 and Table 2.

For most variables, there were no significant difference between lost patients and patients included within the followup analysis. There were also no differences in patients with and without an additional chronic pain entity, which were lost to follow-up ( $\left.p=0.301, \mathrm{X}^{2}=1.737\right)$.

\section{Prevalence of persistent hip pain after hip surgery}

Six months after surgery, $26 \%$ of the patients reported pain in the area of the operated hip ( $>0$ NRS) at the time of the interview, while 58\% reported having had hip pain within the last 4 weeks before the follow-up interview. The median of the reported maximum pain intensity during the last 4 weeks before the follow-up was $2(1.2-4)$ on the NRS. A total of $17 \%(n=18)$ of the 104 patients with pain reports had moderate-to-severe maximum pain intensity ( $>3 \mathrm{NRS}$ ) during the last four weeks before the follow-up interview, whereas $83 \%$ of the patients had no pain or mild pain only ( $\leq 3 \mathrm{NRS})$.

\section{Pre-existing chronic pain entities}

In the follow-up cohort, $69(66 \%)$ patients had at least one chronic pain entity in addition to hip pain at the time of surgery, whereas 35 (34\%) patients had no other pain entity.

There was no statistically significant influence of other pre-existing chronic pain entities beyond the hip with respect to the prevalence or intensity of persistent post-operative hip pain (chronic pain/no chronic pain-prevalence or intensity of persistent postoperative hip defined by: " $>0 \mathrm{NRS}$ " $p=0.275$, $\mathrm{X}^{2}=1.489$; ">3 NRS" $p=0.411, \mathrm{X}^{2}=1.274$; maximum pain intensity during the last four weeks before the follow-up: $p=0.077, Z=-1.769$; hip pain intensity at the time of the follow-up interview: $p=0.189, \mathrm{Z}=-1.314$ ).

\section{Impact of persistent postoperative hip pain intensity on daily life}

There was a significant improvement in the quality of life 6 months after surgery (overall pre vs postoperative mental health [SF 12]: 49.2 $\pm 11.9 / 55.0 \pm 7.5, p=0.011$; pre vs postoperative physical health [SF 12]: 29.9 $\pm 7.5 / 44.4 \pm 10.1, p<0.001$ ), but no differences in values for mental health and physical health status were observed between patients with no or mild persistent postoperative pain $(\mathrm{NRS}<3$ ) vs patients with moderate and severe pain intensity 6 months after surgery (Table 3 ).

Table I Follow-up patient's preoperative general characteristics, group differences, univariate analysis

\begin{tabular}{|c|c|c|c|c|c|}
\hline \multirow[t]{2}{*}{ Variable } & \multirow[t]{2}{*}{ Total $(n=104)$} & \multicolumn{2}{|c|}{$\begin{array}{l}\text { Maximum pain intensity } 6 \text { months } \\
\text { after total hip replacement }\end{array}$} & \multicolumn{2}{|c|}{ Group differences } \\
\hline & & NRS $\leq 3(n=86)$ & NRS >3 $(n=18)$ & $\begin{array}{l}\text { P-values and } \\
{ }^{+} Z \text {-value }{ }^{++} \mathbf{X}^{2}\end{array}$ & \\
\hline \multicolumn{6}{|l|}{ General preoperative characteristics } \\
\hline Age (years) & $63.0 \pm 12.6$ & $62.2 \pm 12,8$ & $66.8 \pm I I . I$ & 0.185 & $1.324^{+}$ \\
\hline Female proportion (\%) & 58 & 59 & 50 & 0.468 & $0.528^{++}$ \\
\hline $\mathrm{BMI}\left(\mathrm{kg} / \mathrm{m}^{2}\right)$ & $28.0 \pm 5.0$ & $27.4 \pm 4.9$ & $30.8 \pm 4.9$ & 0.008 & $2.659^{+}$ \\
\hline Mental health (SF I2) & $49.2 \pm 11.8$ & $49.4 \pm 12.0$ & $48.5 \pm 11.3$ & 0.719 & $0.360^{+}$ \\
\hline Physical health (SF I2) & $29.9 \pm 7.5$ & $30.0 \pm 7.3$ & $29.3 \pm 8.6$ & 0.935 & $0.082^{+}$ \\
\hline \multicolumn{6}{|l|}{ Preoperative pain characteristics } \\
\hline Preoperative maximum hip pain intensity (NRS) & $8.0(7.0-9.0)$ & $8.0(7.0-9.0)$ & $9.0(8.0-9.0)$ & 0.246 & $1.159^{+}$ \\
\hline \multicolumn{6}{|l|}{ Chronic Pain Grade (\%) } \\
\hline I & 16 & 16 & 13 & 0.057 & -1.904 \\
\hline 2 & 25 & 26 & 25 & & \\
\hline 3 & 20 & 22 & 6 & & \\
\hline 4 & 39 & 36 & 56 & & \\
\hline Preoperative analgesic consumption (MQS) & $3.4(0.0-6.5)$ & $3.4(0.0-6.8)$ & $3.4(0.0-5.7)$ & 0.839 & $0.204^{+}$ \\
\hline Neuropathic pain characteristics (painDETECT) & $9.0(4.0-12.25)$. & $8.0(4.0-12.0)$ & II.5 (5.75-| 4.75$)$ & 0.120 & $1.555^{+}$ \\
\hline
\end{tabular}

Notes: Ordinal variables were presented using the median (median [I-3 Quartile]). Metric variables were presented using the arithmetic mean and the SD (mean \pm SD). Abbreviations: BMI, body mass index; MQS, Medication Quantification Scale; NRS, Numeric Rating Scale; SF I2, Short-Form I2. 
Table 2 Follow-up patient's preoperative psychological distress and physical pain testing, group difference, univariate analysis

\begin{tabular}{|c|c|c|c|c|c|}
\hline & \multirow[t]{2}{*}{ Total $(n=104)$} & \multicolumn{2}{|c|}{$\begin{array}{l}\text { Maximum pain intensity } 6 \text { months } \\
\text { after total hip replacement }\end{array}$} & \multicolumn{2}{|c|}{ Group differences } \\
\hline & & NRS $\leq 3(n=86)$ & NRS $>3(n=18)$ & p-value & Z-value \\
\hline \multicolumn{6}{|l|}{ Psychological distress } \\
\hline Depression (DASS) & $3.0(1.0-5.0)$ & $2.0(1.0-5.0)$ & $5(2.25-8.75)$ & 0.032 & 2.144 \\
\hline Anxiety (DASS) & $1.0(0.0-3.0)$ & $1.0(0.0-2.0)$ & $2.5(1.0-3.75)$ & 0.013 & 2.488 \\
\hline Stress (DASS) & $5.0(2.0-8.0)$ & $4.0(1.5-7.0)$ & $6.5(5.0-10.5)$ & 0.038 & 2.074 \\
\hline Kinesiophobia (TSK) & $36.0(32.0-41.0)$ & $35.0(31.0-40.25)$ & $39.0(34.0-44.5)$ & 0.063 & 1.857 \\
\hline Catastrophizing (CTS) & $0.9 \pm 1.2$ & $0.7 \pm 1.0$ & $1.7 \pm 1.6$ & 0.013 & 2.482 \\
\hline Help-/Hopelessness Scale (THS) & $2.1 \pm 1.7$ & $1.9 \pm 1.7$ & $3.3 \pm 1.6$ & 0.003 & 2.927 \\
\hline Thought Suppression (TSS) & $2.7 \pm 1.6$ & $2.5 \pm 1.6$ & $3.8 \pm 1.4$ & 0.004 & 2.900 \\
\hline Somatization (PHQ) & $5.0(4.0-8.0)$ & $5.0(4.0-7.0)$ & $6.0(4.0-8.5)$ & $0.38 \mathrm{I}$ & 0.876 \\
\hline \multicolumn{6}{|l|}{ Physical pain testing } \\
\hline Pressure pain threshold $(\mathrm{kPa})$ & $382.4 \pm 175.0$ & $391.0 \pm 177.8$ & $341.9 \pm 159.0$ & 0.407 & 0.829 \\
\hline Pain inhibition (CPM) & $1.05 \pm 0.19$ & $1.03 \pm 0.16$ & $1.15 \pm 0.26$ & 0.136 & 1.492 \\
\hline
\end{tabular}

Notes: Ordinal variables were presented using the median (median [I-3 Quartile]). Metric variables were presented using the arithmetic mean and the SD (mean \pm SD). Abbreviations: CPM, conditioned pain; CTS, Catastrophizing Thoughts Scale; DASS, Depression Anxiety and Stress Scale; kPa, kilopascals; TSK, Tampa Scale for Kinesiophobia; THS, Thoughts of Help-/Hopelessness Scale; TSS, Thought Suppression Scale; PHQ, patients health questionnaire.

Table 3 Follow-up patient's quality of life, psychological distress, and functionality in daily life activities 6 months after total hip replacement

\begin{tabular}{|c|c|c|c|c|c|}
\hline & \multirow[t]{2}{*}{ Total $(n=104)$} & \multicolumn{2}{|c|}{$\begin{array}{l}\text { Maximum pain intensity } 6 \text { months after } \\
\text { total hip replacement }\end{array}$} & \multicolumn{2}{|c|}{ Group differences } \\
\hline & & NRS $\leq 3(n=86)$ & NRS >3 $(n=18)$ & p-value & Z-value \\
\hline \multicolumn{6}{|l|}{ Quality of life } \\
\hline Mental health (SF I2) & $55.0 \pm 7.5$ & $55.2 \pm 7.5$ & $53.5 \pm 7.4$ & 0.280 & 1.081 \\
\hline Physical health (SF I2) & $44.4 \pm 10.1$ & $44.3 \pm 10.4$ & $44.9 \pm 8.3$ & 0.927 & 0.092 \\
\hline \multicolumn{6}{|l|}{ Psychological distress } \\
\hline Depression (DASS) & $0.0(0.0-3.0)$ & $0.0(0.0-1.0)$ & $4.0(1.75-11)$ & $<0.001$ & 4.487 \\
\hline Anxiety (DASS) & $0.0(0.0-2.0)$ & $0.0(0.0-1.0)$ & $2.0(1.0-7.75)$ & $<0.001$ & 3.776 \\
\hline Stress (DASS) & $\mathrm{I} .0(0.0-3.25)$ & $1.0(0.0-2.0)$ & $6.0(1.0-11.0)$ & 0.002 & 3.097 \\
\hline Kinesiophobia (TSK) & $31.0(28.5-35.0)$ & $30.0(28.5-30.0)$ & $36.5(29.25-43.5)$ & 0.009 & 2.600 \\
\hline \multicolumn{6}{|l|}{ Daily life activity } \\
\hline Disability score (CPG) & $0.0 .(00-0.0)$ & $0.0(0.0-0.0)$ & $3.0(0.0-5.0)$ & $<0.001$ & 4.376 \\
\hline
\end{tabular}

Notes: Ordinal variables were presented using the median (median [I-3 Quartile]). Metric variables were presented using the arithmetic mean and the SD (mean \pm SD). Abbreviations: CPG, Chronic Pain Grade; DASS, Depression Anxiety and Stress Scale; SF 12, Short-Form I2; TSK, Tampa Scale for Kinesiophobia.

Patients with moderate-to-severe pain intensity $(>3$ NRS) 6 months after surgery had more psychological distress (DASS - depression, anxiety, stress; Tampa Scale for Kinesiophobia [TSK] - fear of movement) and more often showed values in the psychological assessment with potential clinical relevance (proportion of patients with relevant score for depression [ $>10$ points] total $8 \%$, "NRS $\leq 3$ " $5 \%$, "NRS $>3$ " $22 \%$, anxiety [ $>6$ points] total $10 \%$, "NRS $\leq 3$ " $6 \%$, "NRS $>3$ " $28 \%$, stress [ $>10$ points] total $10 \%$, "NRS $\leq 3$ " $5 \%$, "NRS $>3$ " $24 \%$; proportion of patients with relevant score for kinesiophobia total [ $>38$ points] $13 \%$, "NRS $\leq 3$ " $11 \%$, "NRS $>3$ " $31 \%$ ). Furthermore, these patients had worse functionality in their daily life activities (CPG - disability points, Table 3 ).

\section{Risk factors for poor functional outcome}

From the univariate analyzes, the variables BMI, preoperative maximum pain intensity (NRS), neuropathic pain characteristics (painDETECT), depression (DASS), helplessness (THS), and the intensity of postoperative pain on movement (NRS) and the maximum intensity of pain postoperatively (NRS) were associated with the functional outcome (disability score of the CPG; Table 4). The multivariate analyses showed that the maximal preoperative pain intensity and the BMI were the only independent factors influencing daily function 6 months after total hip replacement $\left(\mathrm{R}^{2}=0.311, \mathrm{~F}=17.382, p<0.001\right)$.

\section{Discussion}

The current results indicate that a high proportion of patients report some degree of persistent hip pain (maximum hip pain at the follow-up > NRS 0) after total hip replacement. ${ }^{11-14,32}$ The results also indicate that the prevalence of persistent pain (between 26\% and 58\%) has a significant variation depending on the definition adopted. 
Table 4 Univariate analysis of characteristics influencing functionality in daily life activities after 6 months

\begin{tabular}{|c|c|c|}
\hline \multirow[t]{2}{*}{ Variable } & \multicolumn{2}{|c|}{$\begin{array}{l}\text { Univariate analysis on function in daily } \\
\text { life after } 6 \text { months (disability score) }\end{array}$} \\
\hline & $p$-value & Wald \\
\hline Age (years) & 0.078 & 3.10 \\
\hline Sex & 0.264 & 1.249 \\
\hline BMI $\left(\mathrm{kg} / \mathrm{m}^{2}\right)$ & $<0.001$ & 19.18 \\
\hline Mental health (SFI2) & 0.436 & 0.606 \\
\hline Physical health (SFI2) & 0.888 & 0.020 \\
\hline Preoperative maximum hip pain intensity (NRS) & $<0.001$ & 53.763 \\
\hline Preoperative chronic pain grade & 0.009 & 11.351 \\
\hline Preoperative analgesic consumption (MQS) & 0.745 & 0.106 \\
\hline Neuropathic pain characteristics (painDETECT) & $<0.001$ & $|1.64|$ \\
\hline Depression (DASS) & 0.032 & 4.583 \\
\hline Anxiety (DASS) & 0.193 & 1.697 \\
\hline Stress (DASS) & 0.074 & 3.194 \\
\hline Kinesiophobia (TSK) & 0.144 & 2.139 \\
\hline Catastrophizing (CTS) & 0.181 & 1.792 \\
\hline Help-/hopelessness (THS) & 0.039 & 4.246 \\
\hline Thought suppression (TSS) & 0.705 & 0.143 \\
\hline Somatization (PHQ) & 0.597 & 0.279 \\
\hline Pressure pain threshold $(\mathrm{kPa})$ & 0.480 & 0.498 \\
\hline CPM & 0.174 & 1.847 \\
\hline Cumulative postoperative pain on movement (NRS) & 0.017 & 5.748 \\
\hline Cumulative postoperative maximum pain intensity (NRS) & 0.003 & 9.101 \\
\hline Cumulative postoperative opioid consumption (ME) & 0.534 & 0.386 \\
\hline
\end{tabular}

Abbreviations: BMI, body mass index; CPM, conditioned pain modulation; CTS, Catastrophizing Thoughts Scale; DASS, Depression Anxiety and Stress Scale; kPa, kilopascals; ME, Morphine equivalent; MQS, Medication Quantification Scale; NRS, Numeric Rating Scale; SF I2, Short-Form I2; THS, Thoughts of Help-/Hopelessness Scale; TSK, Tampa Scale for Kinesiophobia; TSS, Thought Suppression Scale; PHQ, Patients Health Questionnaire.

However, only a minority of patients report persistent hip pain of moderate or severe intensity (NRS). Persistent pain resulted in a clinically relevant functional impairment (CPG - disability points). Six months after surgery, patients with higher persistent hip pain intensity had more limitations in daily activities (CPG - disability points), and more psychological distress (DASS and TSK). This was not reflected in the patients' concurrent report of their quality of life (SF 12). Nevertheless, there was no difference in self-reported quality of life (SF 12) with respect to pain intensities between patients with low or high pain intensity (NRS). These results raise the question about the clinical relevance of a simple pain report as a potential indicator of functionally relevant persistent pain after hip surgery.

\section{Prevalence of persistent pain}

Depending on the definition used (any pain within the last 4 weeks: NRS $>0$, current pain at the time of the interview NRS $>0$, or moderate-to-severe pain at the time of the interview: NRS $>3$ ), the prevalence of persistent pain after total hip replacement varied widely. Even if 58\% reported having had hip pain within the last 4 weeks before the follow-up interview, only $17 \%$ of all patients reported pain intensities (NRS $>3$ ) considered to be clinically relevant. These results are in line with results from other studies that reported persistent hip pain after total hip replacement in $27 \%-38 \%$ of patients, with $5 \%-12 \%$ of patients reporting moderate or severe pain intensity. ${ }^{2,11-14,32}$ In a large Danish registry of the National Health Service, based on 22,963 patients with total hip replacement, hip pain intensity was rated with a mean of $3.7( \pm 2.5)$ on the NRS, $12-18$ months after surgery in 294 of these patients. In other studies that assessed the maximum pain intensity in two north American cohorts, the median maximum pain intensity after 6 and 12 months was $5 / 10$ and $4 / 10$, respectively. ${ }^{13,32}$ These observations, together with the current results, indicate similarity in different populations of patients following hip joint replacement.

The current study highlights the importance of the definition of persistent pain for determining prevalence. Pain has a dynamic character and it does not appear to be useful to use a simple measure of pain at a single moment in time. These findings emphasize the need for standardization in research on persistent postoperative pain including well-defined time points, frequency, and duration of pain events, which currently differ significantly across studies. ${ }^{9}$ 


\section{Relevance of persistent pain following hip surgery}

Overall, the reported quality of life (SF 12) improved, while the limitations in everyday activities (CPG - disability points) reduced significantly following surgery. Even if patients with higher pain intensity (NRS) had more limitations at the time of the follow-up interview ( $\mathrm{CPG}$ - disability points), this was not reflected in the reported quality of life (SF 12). Nilsdotter Isaksson analyzed the quality of life of patients at 3,6, and 12 months as well as after 4,5 , and 7 years after total hip replacement. Twelve months after surgery, the patients' quality of life reached a plateau of improvement. ${ }^{33}$ Thus, as per these findings, the assessment of persistent pain six months after surgery might still fall into a period of continuing progress in rehabilitation.

These results raise the question of the relevance of a high prevalence of persistent pain after hip surgery, only based on the presence of pain. ${ }^{34}$ It may be more relevant to consider functional impairments related to pain than pain alone.

The reported relevance of neuropathic pain characteristics as a potential marker of nerve damage as a cause of chronic postoperative pain in other surgical procedures could not be confirmed for the current study cohort. These results are consistent with other studies on patients after hip joint surgery. ${ }^{6,11}$ Also in line with other studies, in the current cohort, patients with higher persistent pain intensity (NRS) had greater psychological distress (DASS and TSK). ${ }^{14}$ Nearly one third of the patients had clinically relevant values for symptoms of depression and anxiety, nearly a quarter with "mild" or "severe" pain intensities had clinically relevant values for symptoms of depression and anxiety (DASS). Furthermore, patients with at least moderate persistent pain intensity had more clinically relevant values of fear of movement (TSK). These findings may support the need for the integration of psychological support during rehabilitation after surgery for patients who fall into high-risk groups. ${ }^{18}$ Preoperative pain intensity (NRS) and the BMI were the only independent risk indicators for poor function 6 months after surgery (CPG - disability points). These findings support the assumption of a close relation between physical function and pain. Although preoperative chronic pain has been identified as a relevant factor for slower rehabilitation in the immediate postoperative period, at the current follow-up, there was no effect on reduced function (CPG - disability points) and life quality (SF 12) 6 months after surgery in comparison between patients with and without other chronic pain. ${ }^{18}$

\section{Strengths and limitations}

This was a prospective observational study. The study did not control the standard of clinical care and thus reflects the clinical reality for one clinical center. A strength of this clinical setting was that this hospital adopts a high grade of standardization. For practical reasons, the follow-up was assessed by telephone and the assessment tools used were not formally validated for telephone interviews. However, patients were familiar with the questions from the baseline assessment. Moreover, to exclude personal bias, all telephone interviews were conducted by the same study physician (M.M.), following a standardized protocol. This approach led to a high response rate along with fully completed questionnaires.

The distinction between pre-existing pain and pain initiated by surgery is complex, especially after orthopedic procedures. According to the definition of chronic postoperative pain by Macrae, the important distinction between pre-existing and (new) emerging chronic postoperative pain is indefinite or impossible. ${ }^{7,8}$ In joint surgery, the former definition of chronic pain of the International Association for the Study of Pain (IASP) by Merskey should be reconsidered. Merskey defined chronic pain as a condition that has not regressed within a defined period of 6 months after healing of a tissue defect. ${ }^{35}$ For this reason, in musculoskeletal surgery, from our point of view, the term "persistent" postoperative should be preferred to "chronic" postoperative pain to avoid a possible causal attribution.

The characterization of the patients was very detailed, which is a strength on the one side, and a limitation on the other, due to the sample size in comparison with the number of variables assessed. However, we analyzed risk factors in a two-step approach and only included variables with influence in the univariate analyses. We also reduced variables by summing the respective scores for pain intensity and analgesic consumption. A sample size was not calculated because the sample was defined by the recruitment period.

\section{Conclusion}

Some degree of persistent postoperative pain is a common occurrence after total hip replacement, yet moderate-tosevere pain with functional limitations was rare. However, the prevalence and significance of pain depends on its definition and assessment and should be standardized to ensure comparisons between future studies.

\section{Acknowledgments}

We would like to express our gratitude to Professor Dr. Henning Windhagen and his team at the Department of 
Orthopedic Surgery, Medical School Hannover, Germany, who supported the conduction of the study. We would also like to express our gratitude to our colleague Astrid Degenhart (A.D.) and our research assistants Merle Gathmann (M.G.) and Stephanie Biallas (St.B.).

\section{Author contributions}

All authors contributed toward data collection and interpretation, drafting and critically revising the paper, and agree to be accountable for all aspects of the work.

\section{Disclosure}

The authors report no conflicts of interest in this work.

\section{References}

1. Perkins FM, Kehlet H. Chronic pain as an outcome of surgery. A review of predictive factors. Anesthesiology. 2000;93(4):1123-1133.

2. Fletcher D, Stamer UM, Pogatzki-Zahn E, et al; euCPSP group for the Clinical Trial Network group of the European Society of Anaesthesiology. Chronic postsurgical pain in Europe: An observational study. Eur J Anaesthesiol. 2015;32(10):725-734.

3. Schug S, Pogatzki-Zahn E. Chronic pain after surgery or injury. Pain Clin Updates. 2011;19(1):1-4.

4. Van de Ven TJ, John Hsia HL. Causes and prevention of chronic postsurgical pain. Curr Opin Crit Care. 2012;18(4):366-371.

5. Wildgaard K, Ravn J, Kehlet H. Chronic post-thoracotomy pain: a critical review of pathogenic mechanisms and strategies for prevention. Eur J Cardiothorac Surg. 2009;36(1):170-180.

6. Bonica JJ. The Management of Pain. Philadelphia, London: Lea \& Febiger; 1953.

7. Macrae WA. Chronic pain after surgery. Br JAnaesth. 2001;87(1):88-98.

8. Macrae WA. Chronic post-surgical pain: 10 years on. $B r J$ Anaesth. 2008;101(1):77-86.

9. VanDenKerkhof EG, Peters ML, Bruce J. Chronic pain after surgery: time for standardization? A framework to establish core risk factor and outcome domains for epidemiological studies. Clin J Pain. 2013;29(1):2-8.

10. Werner MU, Kongsgaard UE. I. Defining persistent post-surgical pain: is an update required? Br J Anaesth. 2014;113(1):1-4.

11. Wylde V, Hewlett S, Learmonth ID, Dieppe P. Persistent pain after joint replacement: prevalence, sensory qualities, and postoperative determinants. Pain. 2011;152(3):566-572.

12. Singh JA, Lewallen D. Predictors of pain and use of pain medications following primary Total Hip Arthroplasty (THA): 5,707 THAs at 2-years and 3,289 THAs at 5-years. BMC Musculoskelet Disord. 2010;11:90.

13. Clarke H, Kay J, Mitsakakis N, Katz J. Acute pain after total hip arthroplasty does not predict the development of chronic postsurgical pain 6 months later. J Anesth. 2010;24(4):537-543.

14. Nikolajsen L, Brandsborg B, Lucht U, Jensen TS, Kehlet H. Chronic pain following total hip arthroplasty: a nationwide questionnaire study. Acta Anaesthesiol Scand. 2006;50(4):495-500.

15. Gerbershagen HJ, Ozgur E, Straub K, et al. Prevalence, severity, and chronicity of pain and general health-related quality of life in patients with localized prostate cancer. Eur J Pain. 2008;12(3):339-350.
16. Bruce J, Krukowski ZH. Quality of life and chronic pain four years after gastrointestinal surgery. Dis Colon Rectum. 2006;49(9):1362-1370.

17. Eriksen JR, Poornoroozy P, Jorgensen LN, Jacobsen B, Friis-Andersen HU, Rosenberg J. Pain, quality of life and recovery after laparoscopic ventral hernia repair. Hernia. 2009;13(1):13-21.

18. Erlenwein J, Przemeck M, Degenhart A, et al. The influence of chronic pain on postoperative pain and function after hip surgery: a prospective observational cohort study. J Pain. 2016;17(2):236-247.

19. Erlenwein J, Studer D, Lange JP, Bauer M, Petzke F, Przemeck M. [Process optimization by central control of acute pain therapy: implementation of standardized treatment concepts and central pain management in hospitals]. Anaesthesist. 2012;61(11):971-983. German.

20. Nagel B, Pfingsten M, Lindena G, Nilges P. Deutscher SchmerzFragebogen - Handbuch (German Pain Questionnaire - Manual). Boppard: Deutsche Schmerzgesellschaft; 2012.

21. Racine M, Tousignant-Laflamme Y, Kloda LA, Dion D, Dupuis G, Choiniere M. A systematic literature review of 10 years of research on sex/gender and pain perception - part 2: do biopsychosocial factors alter pain sensitivity differently in women and men? Pain. 2012;153(3): 619-635.

22. Masters Steedman S, Middaugh SJ, Kee WG, Carson DS, Harden RN, Miller MC. Chronic-pain medications: equivalence levels and method of quantifying usage. Clin J Pain. 1992;8(3):204-214.

23. Von Korff M, Ormel J, Keefe FJ, Dworkin SF. Grading the severity of chronic pain. Pain. 1992;50(2):133-149.

24. Schmitt N, Gerbershagen HU. The Mainz pain staging system (MPSS) for chronic pain. Pain. 1990;Suppl 5:484.

25. Freynhagen R, Baron R, Gockel U, Tolle TR. painDETECT: A new screening questionnaire to identify neuropathic components in patients with back pain. Curr Med Res Opin. 2006;22(10):1911-1920.

26. Meissner W, Ullrich K, Zwacka S. Benchmarking as a tool of continuous quality improvement in postoperative pain management. Eur J Anaesthesiol. 2006;23(2):142-148.

27. Sullivan M, Tanzer M, Stanish W, et al. Psychological determinants of problematic outcomes following Total Knee Arthroplasty. Pain. 2009;143(1-2):123-129.

28. Racine M, Tousignant-Laflamme Y, Kloda LA, Dion D, Dupuis G, Choiniere M. A systematic literature review of 10 years of research on sex/gender and experimental pain perception - part 1: are there really differences between women and men? Pain. 2012;153(3):602-618.

29. McNicol ED, Ferguson MC, Hudcova J. Patient controlled opioid analgesia vs non-patient controlled opioid analgesia for postoperative pain. Cochrane Database Syst Rev. 2015;6:CD003348.

30. Grass JA. Patient-controlled analgesia. Anesth Analg. 2005;101(5 Suppl):S44-S61.

31. Hasenbring M. Das Kieler Schmerzinventar (KSI). Bern: Hogrefe Verlag; 1994.

32. Liu SS, Buvanendran A, Rathmell JP, et al. A cross-sectional survey on prevalence and risk factors for persistent postsurgical pain 1 year after total hip and knee replacement. Reg Anesth Pain Med. 2012;37(4):415-422.

33. Nilsdotter $A K$, Isaksson F. Patient relevant outcome 7 years after total hip replacement for OA - a prospective study. BMC Musculoskelet Disord. 2010;11:47.

34. Häuser W, Schmutzer G, Hilbert A, Brahler E, Henningsen P. Prevalence of chronic disabling non - cancer pain and associated demographic and medical variables: a cross-sectional survey in the General German Population. Clin J Pain. 2015;31(10):886-892.

35. Merskey HBN. Classification of chronic pain. Description of chronic pain syndromes and definitions of pain terms. Seattle: IASP-Press; 1994. 


\section{Supplementary material}

Table SI Properties of variables and test

\begin{tabular}{|c|c|c|}
\hline Variable & Characteristic & Properties \\
\hline NRS - Numerical Rating Scale & Pain intensity & $0-10-$ higher number $=$ higher pain intensity \\
\hline MQS - Medication Quantification Scale & $\begin{array}{l}\text { Consumption of analgesics and } \\
\text { coanalgesics }\end{array}$ & $\geq 0$-higher number $=$ more consumption of analgesic \\
\hline CPG - Chronic Pain Grade & Chronic pain-related dysfunction & Grade $0-4$ - no pain/no limiting to high pain/severely limiting \\
\hline MPSS - Mainz Pain Staging System & Classification of chronicity of pain & Stage I-III - higher stage = higher chronicity \\
\hline painDETECT & Neuropathic characteristic of pain & $\begin{array}{l}\text { 0-12 points: unlikely neuropathic component; } \\
\text { 13-18 points: unclear result; } \\
\text { more than } 18 \text { points: probable neuropathic component }\end{array}$ \\
\hline SF 12 - Short-Form 12 & Quality of life & $0-100-$ higher score $=$ better general health \\
\hline $\begin{array}{l}\text { DASS - Depression, Anxiety and Stress } \\
\text { Scale }\end{array}$ & Assessment for psychological distress & $\begin{array}{l}0-21 \text { points }- \text { higher score }=\text { more psychological distress } \\
\text { (for each subscale) }\end{array}$ \\
\hline TSK - Tampa Scale for Kinesiophobia & Pain-related fear of movement & $\begin{array}{l}\text { I7-68 points }- \text { higher score }=\text { higher grade of pain-related fear of } \\
\text { movement }\end{array}$ \\
\hline PHQ - Patient Health Questionnaire & $\begin{array}{l}\text { Somatization of mental health } \\
\text { disorders }\end{array}$ & $\begin{array}{l}\text { 5-9 points: mild; } \\
\text { 10-14 points: moderate; } \\
\text { 15-30 points: severe level of somatization }\end{array}$ \\
\hline CTS* - Catastrophizing Thought Scale & $\begin{array}{l}\text { Tendency of thoughts of } \\
\text { catastrophizing }\end{array}$ & $0-6$ points - higher score $=$ more thoughts of catastrophizing \\
\hline $\begin{array}{l}\text { THS* - Thought of Help-/Hopelessness } \\
\text { Scale }\end{array}$ & Tendency of help-/hopelessness & $0-6$ points - higher score $=$ more thoughts of help-/hopelessness \\
\hline TSS* - Thought of Suppression Scale & $\begin{array}{l}\text { Tendency to suppress negative } \\
\text { thoughts }\end{array}$ & $\begin{array}{l}0-6 \text { points }- \text { higher score }=\text { more tendency to suppress negative } \\
\text { thoughts }\end{array}$ \\
\hline PPT - Pressure Pain Threshold & Overall pain sensitivity & $50-1000 \mathrm{kPa}-$ pressure when felt as a pain (by the patients) \\
\hline CPM - Conditioned Pain Modulation & $\begin{array}{l}\text { Quantification of ability to condition } \\
\text { to a pain }\end{array}$ & $0-I$ : lower values $=$ higher ability of conditioned pain modulation \\
\hline
\end{tabular}

Note: *Subscale of the Kiel Pain Inventory.

Journal of Pain Research

\section{Publish your work in this journal}

The Journal of Pain Research is an international, peer reviewed, open access, online journal that welcomes laboratory and clinical findings in the fields of pain research and the prevention and management of pain. Original research, reviews, symposium reports, hypothesis formation and commentaries are all considered for publication.

\section{Dovepress}

The manuscript management system is completely online and includes a very quick and fair peer-review system, which is all easy to use. Visit http://www.dovepress.com/testimonials.php to read real quotes from published authors. 